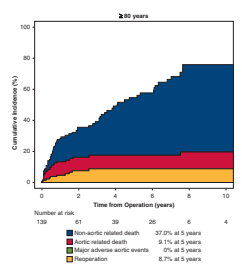

\section{REPLY FROM AUTHORS: WE STILL HAVE MORE TO DO IN OUR LIFE Reply to the Editor:}

In their letter, Harky and colleagues ${ }^{1}$ discuss our article regarding total arch replacement in elderly patients. ${ }^{2}$ They listed their own patients, 24 octogenarians who underwent some thoracic aortic surgery using deep hypothermic circulatory arrest between April 2003 and December 2016. They report elective mortality of $16.7 \%$, stroke rate of $22 \%$, and urgent mortality of $33.3 \%$ for those undergoing deep hypothermic circulatory arrest, with only just more than half being discharged home. Based on their results, they state that elective surgery for thoracic injury in octogenarians is barely acceptable.

Our series consisted of 740 patients who underwent total arch replacement from October 1999 through March 2018. There were 139 patients older than age 80 years old with operative mortality of $8.6 \%$, which is substantially higher than in those undergoing the procedure at a younger age, but it improved over time- to $4.8 \%$ since 2012. Postoperative complications also occurred in more patients in the group aged 80 years or older. There were 52 late deaths, with 4 aortic-related deaths. Survival of the aged 80 years group was $55.4 \%$ at 5 years and $32.2 \%$ at 8 years. Sixtyseven percent of octogenarians or nonagenarians were discharged to home. Multivariable Cox hazard regression analysis demonstrated that chronic kidney disease, nonelective surgery, and concurrent procedures were significant risk factors for overall survival in the group aged 80 years or older. We analyzed the cumulative incidence of adverse outcomes 5 years after total arch replacement in octogenarians or nonagenarians and found that nonaortic-related death was $37.0 \%$, aortic-related death was $9.1 \%$, major adverse aortic events was 0 , and reoperation was $8.7 \%{ }^{2}$

On the other hand, early and late outcomes for elderly patients with acute type A aortic dissection was suboptimal. We analyzed our 328 patients who underwent surgery for acute type A aortic dissection from 1999 to 2016 and found that the hospital mortality showed no difference: $16.1 \%$ in patients aged 75 to 84 years and $11.8 \%$ in patients older than age 85 years. However, only $35 \%$ of patients aged 75 to 84 years and $29 \%$ of patients older than age 85 years went home and follow-up survival at 5 years was $77 \%$ in patients aged 75 to 84 years and $15 \%$ in patients older than age 85 years.

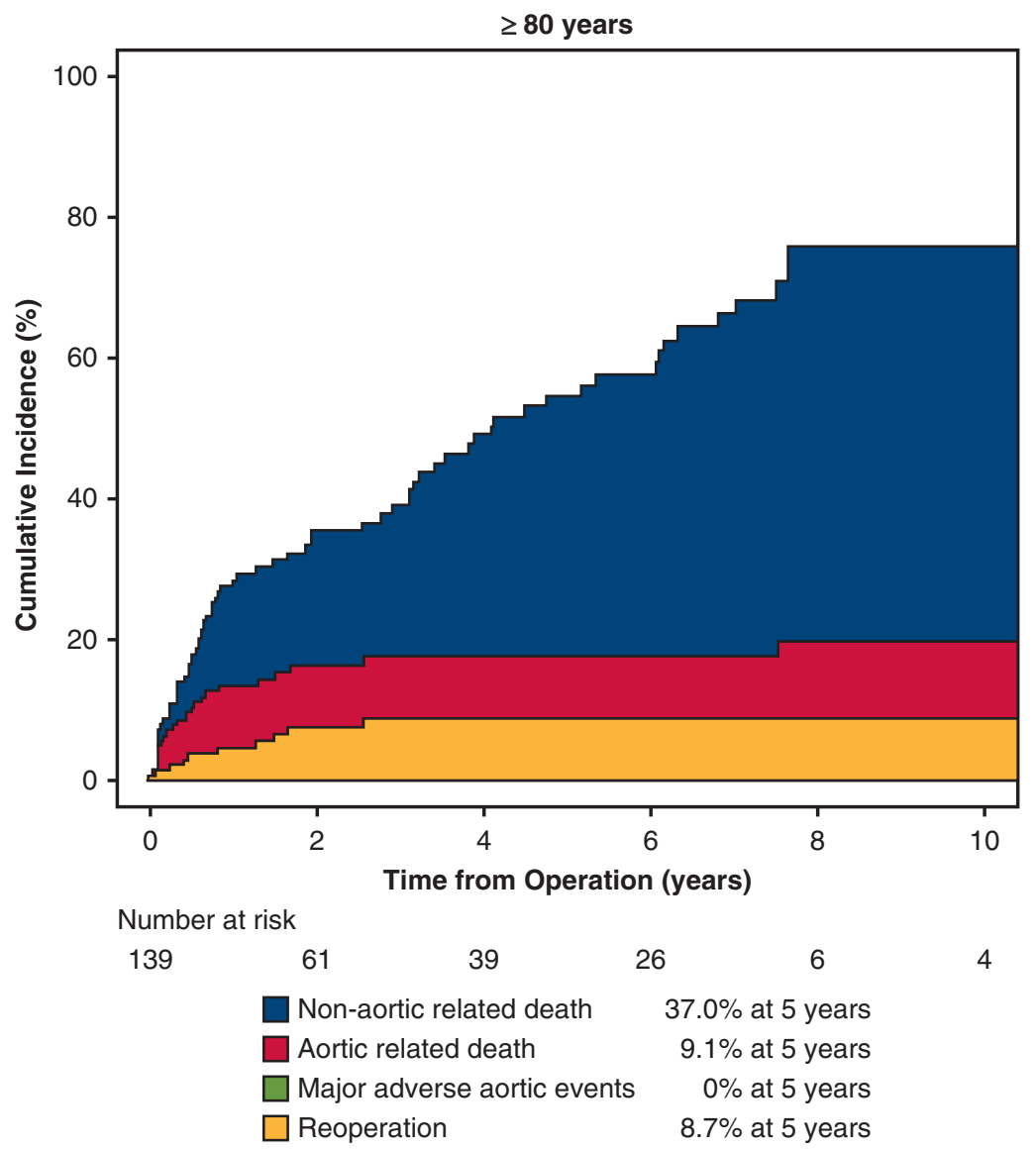

FIGURE 1. Cumulative incidence of adverse outcomes after total arch replacement. 
The authors reported no conflict of interest.

The Journal policy requires editors and reviewers to disclose conflicts of interest and to decline handling or reviewing manuscripts for which they may have a conflict of interest. The editors and reviewers of this article have no conflicts of interest.

In another study of 606 patients who had total arch replacement in our institute, ${ }^{3}$ risk factors for hospital death were older age, low preoperative estimated glomerular filtration rate, organ malperfusion, and longer cardiopulmonary bypass time. Risk factors for new stroke were severe white matter change seen in brain magnetic resonance imaging, ${ }^{4}$ atherosclerotic shaggy aorta, and longer cardiopulmonary bypass time. Risk factors for late death were older age, low preoperative estimated glomerular filtration rate, need for concurrent procedures, permanent neurological deficit, need for tracheostomy, and postoperative acute kidney injury. Our study regarding the frailty assessment ${ }^{5}$ demonstrated that preoperative psoas muscle area index was a good indicator of worse survival after total arch replacement.

Older age is a significant risk factor for early as well as late mortality in total arch replacement. However, postoperative adverse aortic events were very few after total arch replacement even in octogenarians and nonagenarians (Figure 1). We should not reject open surgery simply because of a patient's age and should consider frailty score, sarcopenia score, brain white matter change, or other comorbidities.

Yutaka Okita, MD, PhD ${ }^{a}$
Yuki Ikeno, $M D^{b}$
Kenji Okada, MD, PhD ${ }^{b}$
${ }^{a}$ Cardio-Aortic Center
Takatsuki General Hospital
Osaka, Japan
${ }^{b}$ Division of Cardiovascular Surgery
Department of Surgery
Kobe University
Kobe, Japan

\section{References}

1. Harky A, Bailey G, Othman A, Shaw M, Field M. The life in their years versus the years in their life. J Thorac Cardiovasc Surg. 2021;161:e361-2.

2. Ikeno Y, Yokawa K, Yamanaka K, Inoue T, Tanaka H, Okada K, et al. Total arch replacement in octogenarians and nonagenarians: a single-center 18-year experience. J Thorac Cardiovasc Surg. 2020;160:346-56.e1.

3. Ikeno Y, Yokawa K, Matsueda T, Yamanaka K, Inoue T, Tanaka H, et al. Longterm outcomes of total arch replacement using a 4-branched graft. J Thorac Cardiovasc Surg. 2019;157:75-85.

4. Ikeno Y, Sasaki K, Matsueda T, Inoue T, Tanaka H, Sugimoto K, et al. Impact of white matter changes on neurologic outcomes of total arch replacement using antegrade cerebral perfusion. J Thorac Cardiovasc Surg. 2019;157:1350-7.

5. Ikeno Y, Koide Y, Abe N, Matsueda T, Izawa N, Yamazato T, et al. Impact of sarcopenia on the outcomes of elective total arch replacement in the elderly. Eur J Cardiothorac Surg. 2017;51:1135-41.

https://doi.org/10.1016/j.jtcvs.2020.11.149

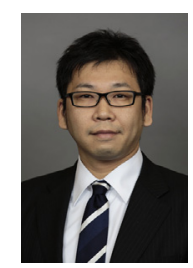

\section{PRIMARY ENTRY CLOSURE IS STILL FIRST-LINE TREATMENT FOR PATIENTS WITH DYNAMIC MALPERFUSION}

To the Editor:

I read with great interest the article by Norton and colleagues, ${ }^{1}$ which reported the efficacy of endovascular fenestration/stenting in patients with acute type $B$ aortic dissection (ATBAD) with malperfusion. In their study cohort, only a limited number $(4.9 \%)$ of ATBAD patients with malperfusion underwent thoracic endovascular aortic repair (TEVAR). ${ }^{1}$ However, I believe that primary entry closure with TEVAR is the first-line treatment for malperfusion, especially due to dynamic occlusion, for the following 2 reasons.

First, in many cases of malperfusion due to dynamic obstruction, TEVAR could provide a quick release of malperfusion with a simple procedure. ATBAD patients with dynamic obstruction often show malperfusion of multiple vascular beds (mesenteric, renal, and iliofemoral), and therefore, rapid relief of malperfusion is critically important in these patients. In fact, 2 patients with malperfusion of multiple organs (case 8 and case 11) died from malperfusion syndrome with severe lower extremity ischemia, as shown in their Table E1. ${ }^{1}$ Although the authors did not provide the procedure time data, it is important to examine the time to resolve malperfusion with their treatment strategy of endovascular fenestration/stenting. Furthermore, the reproducibility of their procedure in centers with less experience needs to be evaluated for their procedure to be accepted as the standard of care.

Second, because the endovascular fenestration/stenting does not close the primary entry, the false lumen pressure is not sufficiently reduced and the high false lumen pressure may lead to aortic rupture. As shown in their Table E1, half of the patients who died (cases 5-7, 9, 10, 12, and 14) died suddenly, ${ }^{1}$ and it is estimated that aortic rupture occurred in the majority. Because dynamic obstruction is caused by increased false lumen pressure, ATBAD patients with dynamic obstruction are believed to be at higher risk for aortic rupture. Therefore, primary entry closure with TEVAR could lower the risk of aortic rupture by decreasing the false lumen pressure.

Of course, I agree with the authors' assertion that endovascular fenestration/stenting is recommended in ATBAD 